\title{
Risk of mortality with concomitant use of tamoxifen and selective serotonin reuptake inhibitors: multi-database cohort study
}

\author{
Macarius M Donneyong, ${ }^{1}$ Katsiaryna Bykov, ${ }^{1,2}$ Pauline Bosco-Levy, ${ }^{1}$ Yaa-Hui Dong,, Raisa Levin, \\ Joshua J Gagne $e^{1,2}$
}

${ }^{1}$ Division of

Pharmacoepidemiology and

Pharmacoeconomics,

Department of Medicine,

Brigham and Women's Hospital

and Harvard Medical School,

Boston, MA, USA 02120

2Department of Epidemiology,

Harvard T.H. Chan School of

Public Health, Boston, MA, USA

02115

Correspondence to: J J Gagne jgagne1@partners.org

Additional material is published online only. To view please visit the journal online.

Cite this as: BMJ 2016;354:i5014 http://dx.doi.org/10.1136/bmj.i5014

Accepted: 12 September 2016

\begin{abstract}
OBJECTIVE

To compare differences in mortality between women concomitantly treated with tamoxifen and selective serotonin reuptake inhibitors (SSRIs) that are potent inhibitors of the cytochrome-P450 2D6 enzyme (CYP2D6) versus tamoxifen and other SSRIs.

DESIGN

Population based cohort study.

SETTING

Five US databases covering individuals enrolled in private and public health insurance programs from 1995 to 2013.
\end{abstract}

\section{PARTICIPANTS}

Two cohorts of women who started taking tamoxifen. In cohort 1, women started taking an SSRI during tamoxifen treatment. In cohort 2, women were already taking an SSRI when they started taking tamoxifen.

\section{MAIN OUTCOME MEASURES}

All cause mortality in each cohort in women taking SSRIs that are potent inhibitors of CYP2D6 (paroxetine, fluoxetine) versus other SSRIs. Propensity scores were used to match exposure groups in a variable ratio fashion. Results were measured separately for each cohort and combined hazard ratios calculated from Cox regression models across the two cohorts with random effects meta-analysis.

RESULTS

There were 6067 and 8465 new users of tamoxifen in cohorts 1 and 2, respectively. Mean age was 55. A total of 991 and 1014 deaths occurred in cohorts 1 and 2 during a median follow-up of 2.2 (interquartile range $0.9-4.5)$ and $2.0(0.8-3.9)$ years, respectively. The pooled hazard ratio for death for potent inhibitors (rate $58.6 / 1000$ person years) compared with other SSRIs (rate 57.9/1000 person years) across cohorts 1 and 2

\section{WHAT IS ALREADY KNOWN ON THIS TOPIC}

Nearly half of women with breast cancer report depression, anxiety, or both, which are associated with an increased risk of mortality

Tamoxifen, a prodrug that reduces recurrence of breast cancer and associated mortality, is activated by the cytochrome-P450 (CYP) enzyme (CYP2D6)

The selective serotonin reuptake inhibitors (SSRIs), paroxetine and fluoxetine, are potent inhibitors of CYP2D6 and could theoretically reduce the bioavailability of the main tamoxifen active metabolite, which could reduce the its effectiveness

\section{WHAT THIS STUDY ADDS}

In a large population of women treated with tamoxifen, use of paroxetine and fluoxetine versus SSRIs that do not inhibit CYP2D6 did not increase the risk of death SSRI inhibition of CYP2D6 does not seem to reduce the effectiveness of tamoxifen

was 0.96 (95\% confidence interval 0.88 to 1.06 ). Results were consistent across sensitivity analyses.

\section{CONCLUSION}

Concomitant use of tamoxifen and potent CYP2D6 inhibiting SSRIs versus other SSRIs was not associated with an increased risk of death.

\section{Introduction}

Breast cancer is the most common invasive cancer and is the leading cause of cancer related mortality among women worldwide. ${ }^{1}$ One in every four diagnoses of cancer among women is for breast cancer, and breast cancer accounts for $15 \%$ of cancer deaths in women. ${ }^{2}$ Nearly half of women with breast cancer report depression, anxiety, or both in the first year after diagnosis. Some studies have found higher mortality rates in patients with breast cancer and depression compared with those without depression. ${ }^{34}$

Tamoxifen is a selective estrogen receptor modulator that reduces the risk of recurrence of breast cancer by about half in women with hormone receptor positive tumors (which account for about two thirds of all breast cancers), reduces the risk of breast cancer mortality by about a third, and reduces the risk of all cause mortality by about $22 \% .^{56}$ A prodrug, tamoxifen is converted by the cytochrome P450 (CYP) enzyme system into two active metabolites. ${ }^{7}$ Conversion of tamoxifen to endoxifen, the more important metabolite, is mediated by CYP2D6. ${ }^{8}$ About a quarter of women using tamoxifen also take selective serotonin reuptake inhibitors (SSRIs), and the SSRIs, paroxetine and fluoxetine, are potent inhibitors of CYP2D6. ${ }^{9}$ Concern has been raised that, by inhibiting CYP2D6, these drugs might reduce the bioavailability of endoxifen, which could reduce tamoxifen's effectiveness. ${ }^{10}$

Little evidence is available to guide decisions about treatment with SSRIs in women with breast cancer. One observational study reported increasing rates of death from breast cancer and all cause mortality with increasing duration of concomitant exposure to tamoxifen and paroxetine; no such association was observed for fluoxetine, however, which is also a potent inhibitor of CYP2D6. ${ }^{11}$ The evidence on the effect of concomitant exposure to tamoxifen and SSRIs, especially those that are potent inhibitors of CYP2D6 enzyme activity, remains inconclusive.

We compared differences in mortality between women concomitantly treated with tamoxifen and SSRIs that are potent inhibitors of CYP2D6 (paroxetine, fluoxetine) versus women treated with tamoxifen plus other SSRIs. 


\section{Methods \\ Data sources and settings}

We assembled data from five US electronic healthcare databases, covering individuals who received health insurance coverage from commercial and public payors from 1995 to 2013. Specifically, we used the Optum Research Database (2004-13), which comprises data on UnitedHealth enrollees; Medicare data linked to pharmacy claims data from CVS Caremark (2005-08), the Pennsylvania Pharmaceutical Assistance Contract for the Elderly (PACE; 1995-2005), and the New Jersey Pharmaceutical Assistance to the Aged and Disabled (PAAD; 19952005); and data from the Medicaid Analytic Extract (MAX; 2001-06) covering Medicaid beneficiaries in 49 states and the District of Columbia. Medicare provides health insurance to older Americans (age 65 and older) and to those with certain disabilities, and Medicaid provides insurance benefits to low income individuals and families. Each database contains data on demographic and enrollment records, inpatient and outpatient diagnoses and procedures, outpatient pharmacy dispensing, and death information. Combined, these databases represent each of the three main insured segments of the US population.

\section{Participants and study design}

We identified all women in each database who started taking tamoxifen. We defined initiation by requiring that women have at least 180 days of tamoxifen-free continuous enrollment in the database before their first prescription for tamoxifen. We further restricted the study population to those who were concomitantly exposed to an SSRI. We formed two separate cohorts based on whether women initiated the SSRI after tamoxifen initiation (cohort 1) or whether they had an active SSRI prescription at the time of tamoxifen initiation (cohort 2) (fig 1).12 We defined an active SSRI prescription as a dispensation before the tamoxifen initiation and with a number of days' supply that overlapped the date of tamoxifen initiation. We excluded from both cohorts those women who used aromatase inhibitors before the date of tamoxifen initiation.

\section{Patient involvement}

Our study was a secondary data analysis and did not include patients as study participants. No patients were
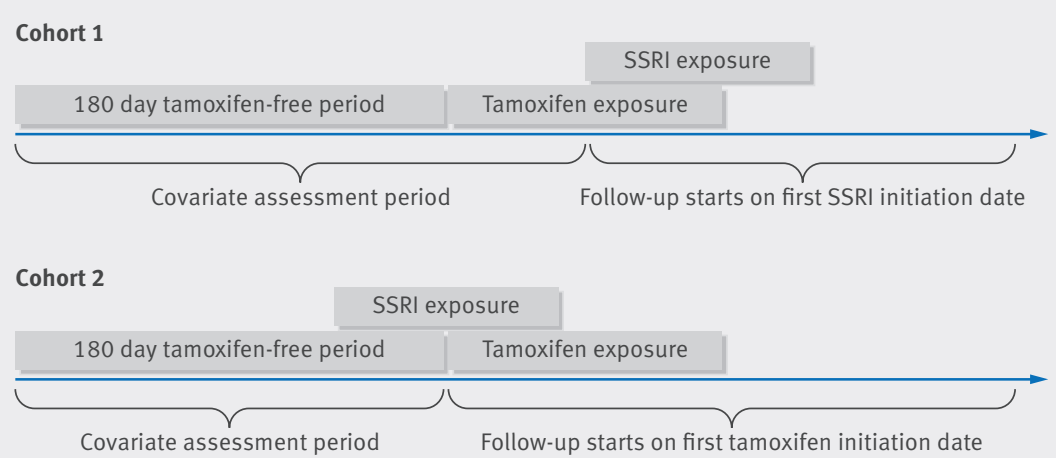

Fig 1 | Descriptions of cohorts of women with breast cancer according to initiation of SSRIs and tamoxifen involved in setting the research question or the outcome measures, nor were they involved in the design and implementation of the study. There are no plans to involve patients in dissemination.

\section{Exposure}

SSRIs were classified as those that are potent inhibitors of CYP2D6 (paroxetine and fluoxetine) and those that are not potent inhibitors of CYP2D6 (citalopram, escitalopram, fluvoxamine, and sertraline). In each cohort, patients were considered as exposed if they received tamoxifen and a potent CYP2D6 inhibiting SSRI and were considered unexposed if they received tamoxifen and another SSRI.

\section{Outcome and follow-up}

We compared rates of all cause mortality between the two exposure groups. All cause mortality was ascertained from data provided by Medicare for patients enrolled in Caremark, PAAD, and PACE and from Medicaid for patients enrolled in MAX. For the Optum Research Database, we ascertained death status through linkage to the Death Master File from the Social Security Administration. Follow-up for all cause mortality started on the day after the first date of concomitant tamoxifen and SSRI exposure (that is, the index date), corresponding to the SSRI initiation date for cohort 1 and the tamoxifen initiation date for cohort 2 . In the main analysis, we allowed women to contribute person time until the first of death, disenrollment from the health plan, or end of available data during follow-up. In an "as treated" sensitivity analysis, we censored women at the earliest of death, disenrollment from the health plan, end of data availability, switching between SSRI groups, or discontinuation of either drug (allowing a 14 day gap between days' supply of consecutive dispensing).

\section{Covariates}

Covariates were assessed during a baseline period before each woman's index date. The baseline period in cohort 1 was defined as the 180 days before tamoxifen initiation plus the days between the dates of tamoxifen and SSRI initiation (fig 1). In cohort 2, the baseline period was defined as the 180 days before the date of tamoxifen initiation (fig 1). We used Medical claims from the baseline period to assess the stage, site, and previous screening and treatments for breast cancer (see appendix 1 for breast cancer diagnoses and procedure codes). We determined the duration of tamoxifen use before the index date for those in cohort 1 and the numbers of days since the most recent SSRI dispensing before the index date for those in cohort 2. Use of prescribed drugs, including other drugs used to treat depression and anxiety and drugs for conditions associated with mortality, such as cardiovascular diseases, was also measured. We also assessed exposure to other drugs that affect the CYP2D6 enzyme, including both inducers and inhibitors (see appendix 1). Chronic disease burden was measured with the combined Charlson index and the Elixhauser measure score, which 
summarizes 20 clinical conditions. ${ }^{13}$ Measures of use of healthcare, including numbers of physician visits, days spent in hospital, and dispensed drugs were also assessed during the baseline period.

\section{Statistical analysis}

We used propensity scores to summarize the covariates listed above. Propensity scores, the probability of being exposed to potent CYP2D6 inhibiting SSRIs versus other SSRIs, were estimated separately for each database. Exposed and unexposed groups were matched on the propensity scores in a variable ratio of up to 1:10. That is, we used a nearest neighbor algorithm to match exposed individuals to up to 10 unexposed individuals within a caliper of 0.025 on the propensity score scale. This enabled us to leverage the larger group of unexposed patients while still realizing the benefits of propensity score matching.

The distributions of baseline covariates were compared between exposed and unexposed groups in both unmatched and matched cohorts in each database and in the total population of patients across all five databases. To assess covariate balance in the variable ratio matched cohort, we randomly selected one unexposed individual from each set of women matched to an exposed individual, yielding a sample of unexposed individuals of the same size as the exposed group, which permits direct comparison of covariate distributions between groups. ${ }^{14}$

We performed propensity score matching and effect estimation separately within each cohort and within each database. Cox proportional hazards models, stratified on the matching ratio, were used to estimate hazard ratios and 95\% confidence intervals. We then combined the results between cohorts 1 and 2 and across databases using a random effects meta-analysis. We quantified statistical heterogeneity across the estimates using $\mathrm{I}^{2}$.

\section{Sensitivity analyses}

In the as treated analysis, we estimated the effect of concomitant exposure to tamoxifen and SSRIs on mortality only while women were exposed to both drugs during follow-up. We also repeated the primary analysis in those concomitantly exposed to tamoxifen and SSRIs for at least 30, 60, 90, 120, 180, and 240 days; in these analyses, follow-up began at 30, 60, 90, 120, 180, and 240 days, respectively, after the first day of concomitant tamoxifen and SSRI exposure. We carried out these analyses to assess the presence of a potential exposure duration-response effect. In separate subgroup analyses, we repeated the main, as treated, and duration of concomitant exposure analyses among the subpopulation of women with a diagnosis of breast cancer recorded during the baseline period. We also examined paroxetine and fluoxetine as separate exposure groups. To do this, we re-estimated propensity scores predicting paroxetine use versus use of SSRIs that are not potent CYP2D6 inhibitors and predicting fluoxetine use versus use of SSRIs that are not potent CYP2D6 inhibitors and separately re-matched patients and estimated hazard ratios and 95\% confidence intervals in these matched cohorts. Lastly, given that the American Society of Clinical Oncology (ASCO) guidelines recommend tamoxifen as standard adjuvant endocrine treatment for breast cancer among premenopausal women, ${ }^{15}$ we examined potential effect modification by age (that is, $\leq 50$ and $>50$ ) by including an exposure-byage product term in the Cox regression model. Unless otherwise noted, sensitivity analyses were performed with the same statistical methods applied in the main analyses.

\section{Results}

Patients' characteristics

We identified 6067 women who were new users of tamoxifen in cohort 1 and subsequently became concomitantly exposed to SSRIs; 2268 were exposed to potent CYP2D6 inhibiting SSRIs and 3799 were exposed to other SSRIs. In cohort 2, we identified 8465 new users of tamoxifen who were already active users of SSRIs at the time of tamoxifen initiation; 3531 were users of potent CYP2D6 inhibiting SSRIs and 4934 were exposed to other SSRIs. Table 1 summarizes the characteristics of patients in each cohort for both unmatched and propensity score matched groups (complete list of covariates is provided in table $\mathrm{A}$, appendix 2). There were no major differences in patients' characteristics between the two exposure groups even before matching patients. In cohort 1, the matched groups included 2026 users of potent CYP2D6 inhibiting SSRIs (1205 paroxetine and 821 fluoxetine) and 3774 users of other SSRIs (934 citalopram, 1121 escitalopram, 19 fluvoxamine, and 1649 sertraline). In cohort 2, the matched groups included 3025 users of potent inhibiting SSRIs (1653 paroxetine and 1372 fluoxetine) and 4823 users of other SSRIs (1205 citalopram, 1329 escitalopram, 33 fluvoxamine, and 2201 sertraline). Matching further reduced differences in observed covariates between exposure groups in both cohorts. The mean age of patients in cohorts 1 and 2 was 56 (SD 14) and 55 (SD 13), respectively. Overall, 57\% of the potent CYP2D6 inhibiting SSRI group used paroxetine. About half of the patients in both cohorts had a diagnosis code for breast cancer recorded in the 180 days before tamoxifen initiation; more than half of these diagnoses were for stage 0 or I disease. On average, patients in cohort 1 used tamoxifen for 148 days before their first SSRI exposure.

\section{Main results}

During a median follow up of 2.2 (interquartile range 0.9-4.5) years, 991 patients in cohort 1 died at a rate of 64.3 deaths (95\% confidence interval 60.4 to 68.4) per 1000 person years. In cohort 2, there were 1014 deaths over a median follow-up of 2.0 (0.8-3.9) years, for a rate of 53.3 (50.1 to 56.7) per 1000 person years. Table B in appendix 2 shows crude results before propensity score matching. Figure 2 shows the hazard ratios for death stratified by each database and cohort after adjustment with propensity score matching. The pooled rates of death were 58.6/1000 person years among users of SSRIs that are potent inhibitors and 57.9/1000 person 


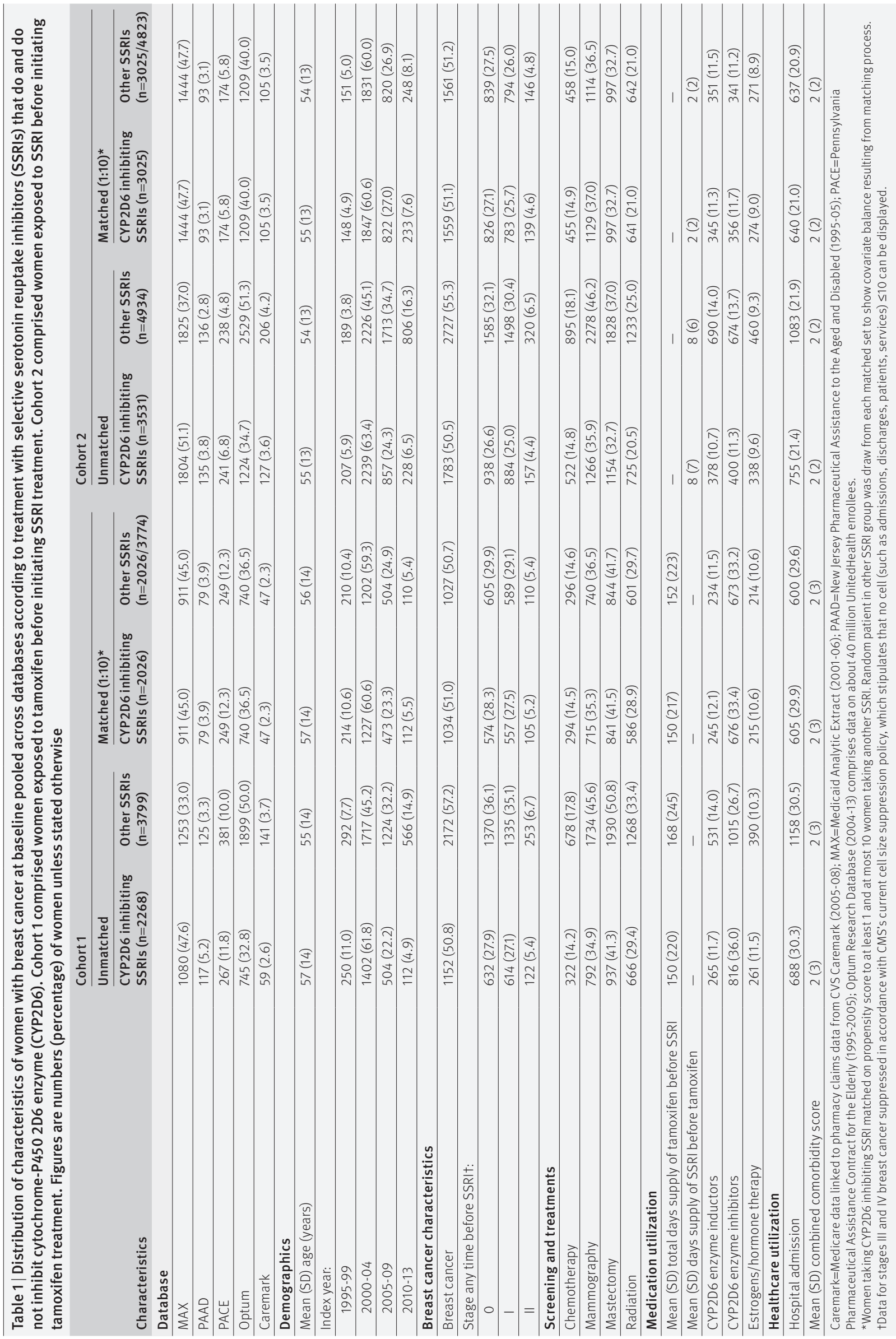




\begin{tabular}{|c|c|c|c|c|}
\hline $\begin{array}{l}\text { Subgroup } \\
\text { Cohort } 1 \text { (SSRI initiated after tamox }\end{array}$ & Cohort 1 (SSRI initiated after tamoxifen) & \multicolumn{2}{|c|}{$\begin{array}{l}\text { Hazard ratio } \\
(95 \% \mathrm{Cl})\end{array}$} & $\begin{array}{l}\text { Hazard ratio } \\
\quad(95 \% \mathrm{CI})\end{array}$ \\
\hline CAREMARK & $46 / 174$ & & & $0.94(0.45$ to 2.00$)$ \\
\hline MAX & $126 / 2112$ & & & $0.95(0.67$ to 1.33$)$ \\
\hline PACE & $509 / 624$ & & $\rightarrow$ & 0.81 (0.67 to 0.97$)$ \\
\hline PAAD & $115 / 201$ & & & $1.15(0.77$ to 1.72$)$ \\
\hline UNITED & $195 / 2638$ & & $\rightarrow$ & $0.93(0.69$ to 1.27$)$ \\
\hline Pooled databases (cohort 1) & $991 / 5749$ & & $\rightarrow$ & $0.91(0.80$ to 1.04$)$ \\
\hline \multicolumn{5}{|c|}{ Cohort 2 (tamoxifen initiated after SSRI) } \\
\hline CAREMARK & $101 / 307$ & & & $0.69(0.41$ to 1.15$)$ \\
\hline $\operatorname{MAX}$ & $209 / 3113$ & & & $1.04(0.79$ to 1.36$)$ \\
\hline PACE & $322 / 410$ & & - & $0.99(0.78$ to 1.25$)$ \\
\hline PAAD & $122 / 226$ & & & $1.16(0.80$ to 1.68$)$ \\
\hline UNITED & $260 / 3737$ & & 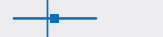 & $1.04(0.80$ to 1.36$)$ \\
\hline Pooled databases (cohort 2) & $1014 / 7793$ & & $\longrightarrow$ & $1.02(0.89$ to 1.16$)$ \\
\hline \multirow[t]{3}{*}{ Total population (cohorts 1 and 2) } & $2005 / 13542$ & & $\rightarrow$ & $0.96(0.88$ to 1.06$)$ \\
\hline & 0.36 & 0.60 & 1.00 & 2.70 \\
\hline & $\begin{array}{l}\text { Lower } \\
\text { of mo }\end{array}$ & $\begin{array}{l}\text { risk } \\
\text { tality }\end{array}$ & $\begin{array}{l}\text { High } \\
\text { of mo }\end{array}$ & $\begin{array}{l}\text { er risk } \\
\text { tality }\end{array}$ \\
\hline
\end{tabular}

Fig 2 | Risk of all cause mortality associated with CYP2D6 inhibiting selective serotonin reuptake inhibitors (SSRIs) versus other SSRIs in main analyses in women exposed to tamoxifen before initiation of SSRI (cohort 1) and women exposed to SSRI before initiation of tamoxifen (cohort 2). Caremark=Medicare data linked to pharmacy claims data from CVS Caremark (2005-08); MAX=Medicaid Analytic Extract (2001-06); PAAD=New Jersey Pharmaceutical Assistance to the Aged and Disabled (1995-2005); PACE=Pennsylvania Pharmaceutical Assistance Contract for the Elderly (1995-2005); Optum Research Database (2004-13) comprises data on approximately 40 million UnitedHealth enrollees $\left(I^{2}=0 \%\right.$ for heterogeneity between databases) years for users of other SSRIs. When we pooled the hazard ratios across databases within each cohort, concomitant exposure to tamoxifen and SSRIs that inhibit CYP2D6 was not associated with an increase in all cause mortality compared with concomitant exposure to tamoxifen and other SSRIs in either cohort 1 (hazard ratio $0.91,0.80$ to 1.04 ) or cohort 2 (1.02, 0.89 to 1.16$)$. The hazard ratio for the combined pooled estimates for both cohorts was 0.96 ( 0.88 to 1.06). There was no statistical heterogeneity in any of the pooled estimates ( ${ }^{2}<1 \%$ for all analyses).

\section{Sensitivity analyses}

In the as treated analysis in which patients were censored when they switched between SSRI groups or when they discontinued treatment with either tamoxifen or an SSRI, the overall pooled estimate was similar to that of the primary analysis estimate (hazard ratio $0.99,95 \%$ confidence interval 0.67 to $1.46 ; \mathrm{I}^{2}=0 \%$; table 2 and table $\mathrm{C}$ in appendix 2). When we stratified the primary analysis by length of concomitant exposure, the pooled hazard ratios were similar to the primary analysis results (fig 3). We found no differences in mortality among patients concomitantly exposed to tamoxifen and paroxetine versus SSRIs that are not potent inhibitors of CYP2D6 or among those concomitantly exposed to fluoxetine versus SSRIs that are not potent inhibitors

\begin{tabular}{|c|c|c|c|c|}
\hline Study design & Deaths/total & Person years & Rate* & $\mathrm{HR}(95 \% \mathrm{Cl})$ \\
\hline \multicolumn{5}{|l|}{ Primary/main analysis } \\
\hline Pooled databases (cohort 1) & $991 / 5749$ & 15419 & 64.3 & $0.91(0.80$ to 1.04$)$ \\
\hline Pooled databases (cohort 2) & $1014 / 7793$ & 190210 & 53.3 & $1.02(0.89$ to 1.16$)$ \\
\hline Overall population (cohorts 1 and 2) & 2005/13542 & 34448 & 58.2 & 0.96 (0.88 to 1.06$)$ \\
\hline \multicolumn{5}{|l|}{ Sensitivity analyses } \\
\hline \multicolumn{5}{|l|}{ As treated analysis $¥$} \\
\hline Pooled databases (cohort 1) & 39/5749 & 1751 & 22.2 & 0.68 (0.34 to 1.38$)$ \\
\hline Pooled databases (cohort 2) & $77 / 7793$ & 3273 & 23.5 & $1.19(0.75$ to 1.89$)$ \\
\hline Overall population (cohorts 1 and 2) & $116 / 13542$ & 5024 & 23.0 & 0.99 (0.67 to 1.46$)$ \\
\hline \multicolumn{5}{|c|}{ Patients with pre-existing breast cancer diagnosis (stages 0-IV) } \\
\hline Pooled databases (cohort 1) & 429/3071 & 7571 & 56.7 & 0.99 (0.81 to 1.21$)$ \\
\hline Pooled databases (cohort 2) & $460 / 4199$ & 9779 & 47.0 & 1.07 (0.88 to 1.31$)$ \\
\hline Overall population (cohorts 1 and 2) & $889 / 7270$ & 17350 & 51.2 & 1.03 (0.89 to 1.18$)$ \\
\hline \multicolumn{5}{|c|}{ Patients with pre-existing breast cancer diagnosis (stages I-IV only) } \\
\hline Pooled databases (cohort 1) & $316 / 1760$ & 4472 & 70.7 & 0.95 (0.75 to 1.21$)$ \\
\hline Pooled databases (cohort 2) & $346 / 2524$ & 6059 & 57.1 & 1.07 (0.85 to 1.33$)$ \\
\hline Overall population (cohorts 1 and 2) & $662 / 4284$ & 10530 & 62.9 & 1.04 (0.86 to 1.26$)$ \\
\hline \multicolumn{5}{|l|}{ Paroxetine use $v$ other SSRIs§ } \\
\hline Pooled databases (cohort 1) & $312 / 3936$ & 9327 & 33.5 & 1.03 (0.80 to 1.33$)$ \\
\hline Pooled databases (cohort 2) & $466 / 5824$ & 13671 & 34.1 & 0.89 (0.72 to 1.11$)$ \\
\hline Overall population (cohorts 1 and 2) & $778 / 9760$ & 22998 & 33.8 & 0.95 (0.81 to 1.12$)$ \\
\hline \multicolumn{5}{|l|}{ Fluoxetine use $v$ other SSRIs } \\
\hline Pooled databases (cohort 1) & $284 / 4047$ & 9180 & 30.9 & $0.99(0.73$ to 1.34$)$ \\
\hline Pooled databases (cohort 2) & $458 / 5685$ & 13178 & 34.8 & 1.05 (0.85 to 1.31$)$ \\
\hline Overall population (cohorts 1 and 2) & $742 / 9735$ & 22358 & 33.2 & 1.04 (0.87 to 1.25$)$ \\
\hline
\end{tabular}

\section{*Rate per 1000 person years.}

†All sensitivity analyses used main effects analytic approach, except for analysis explicitly described as "as treated" sensitivity analysis.

$\ddagger$ Patients censored at earliest of death, disenrollment from health plan, end of data availability, switching between SSRI groups, or discontinuation of either drug (allowing 14 day gap between days' supply of consecutive dispensing).

$\S$ Excluding fluoxetine users.

१Excluding paroxetine users. 


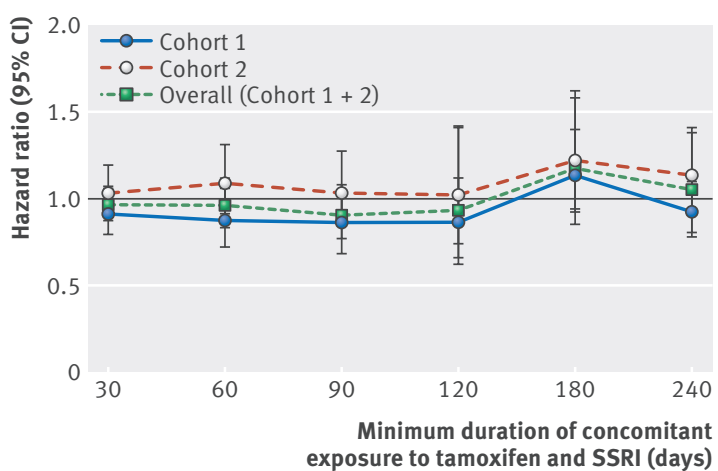

Fig 3 | Risk of all cause mortality associated with CYP2D6 inhibiting selective serotonin reuptake inhibitors (SSRIs) versus other SSRIs stratified by duration of concomitant exposure to tamoxifen and SSRIs before follow-up. Caremark=Medicare data linked to pharmacy claims data from CVS Caremark (2005-08); MAX=Medicaid Analytic Extract (2001-06); PAAD=New Jersey Pharmaceutical Assistance to the Aged and Disabled (1995-2005); PACE=Pennsylvania Pharmaceutical Assistance Contract for the Elderly (1995-2005); Optum Research Database (2004-13) comprises data on approximately 40 million UnitedHealth enrollees

of CYP2D6 (table 2). Results were also similar in the subpopulation of women with a diagnosis of breast cancer (stages 0-IV) recorded in the 180 days before tamoxifen initiation (1.03, 0.89 to $1.18 ; \mathrm{I}^{2}=0 \%$; table 2 and table D in appendix 2). When this subpopulation of patients with breast cancer was further restricted to only those with stages I-IV breast cancer the hazard ratio was 1.04 ( 0.86 to 1.26 ; $\mathrm{I}^{2}=0 \%$; table 2 ). Finally, we observed significant effect modification between age $(\leq 51 v>51)$ and the type of SSRI (that is, potent CYP2D6 inhibitors $v$ other SSRIs) on mortality ( $\mathrm{P}=0.01$ for interaction). Neither stratified estimate, however, was different from the null (1.19 (0.93 to 1.52) for age $\leq 51$ and 0.93 (0.84 to 1.03) for age $>51$ ). Additional database-specific results are provided in appendix 2 .

\section{Discussion}

\section{Principal findings}

In a large cohort of 14532 women who were new users of tamoxifen with a mean age of about 55 enrolled in US commercial and public health insurance plans, we found no difference in rates of all cause mortality between those exposed to SSRIs that are potent inhibitors of the CYP2D6 enzyme compared with those exposed to other SSRIs. This finding was consistent across sensitivity analyses and in analyses examining the relation between the duration of concomitant exposure to tamoxifen and SSRIs and mortality.

Our findings are consistent with a meta-analysis of observational studies that reported no increase in mortality with concomitant exposure to tamoxifen and SSRIs that were either weak (hazard ratio 1.05, 95\% confidence interval 0.91 to 1.22 ) or strong (1.03, 0.86 to 1.23 ) inhibitors of CYP2D6. ${ }^{16}$ The largest study in the meta-analysis, which included 2430 women with breast cancer treated with tamoxifen, found an increased risk of all cause and breast cancer specific mortality with concomitant exposure to paroxetine but not fluoxetine ${ }^{11}$; neither SSRI was associated with greater mortality among women concurrently using tamoxifen in our study. Our study sample was much larger and looked directly at women treated with tamoxifen who were also exposed to SSRIs that are strong inhibitors of CYP2D6 compared with women exposed to other SSRIs. Also, women included in our analyses were relatively younger (mean (SD) age 54.7 (13.2)) and might therefore have had lower prevalence of morbidity and a lower risk of mortality than the women in the previous study, who had a mean age of $77 .{ }^{11}$

\section{Clinical implications}

Our findings suggest that the inhibition of CYP2D6 enzyme function by SSRIs might not impair the effectiveness of tamoxifen in reducing the risk of all cause mortality. While we were not able to measure the concentration of tamoxifen metabolites, even if SSRIs that are strong CYP2D6 inhibitors lowered plasma concentrations of tamoxifen metabolites by a third, as previously reported, these metabolites would still be in high enough concentration to competitively inhibit the estrogen receptor positive cells from getting the estrogen required for growth. ${ }^{17-19}$ Indeed, the usual tamoxifen dose of $20 \mathrm{mg} /$ day is about 20 times higher than the doses needed to be clinically effective. ${ }^{18}$ Furthermore, even if the plasma concentrations of endoxifen, the major active metabolite of tamoxifen, were lowered, other metabolites could still confer sufficient antiestrogenic activity against tumor cells to reduce mortality.

It is also plausible that the degree by which tamoxifen is rendered ineffective by CYP2D6 inhibition varies by menopausal status. ${ }^{16}$ Given that the level of estrogen production is higher in premenopausal women, it is believed that a reduction in the plasma concentration of tamoxifen metabolites would compromise the effectiveness of tamoxifen to compete for estrogen and suppress tumor growth. ${ }^{16}$ Our interaction analysis suggested that age is a potential effect modifier of the association between concomitant exposure to tamoxifen and CYP2D6 inhibiting SSRIs and mortality. Further studies powered to detect subgroup findings and with direct information on menopause status, rather than age as a proxy, could further elucidate this finding.

\section{Strengths}

Our study has several important strengths. First, we compared the risk of death in women taking SSRIs that are potent inhibitors and women taking other SSRIs in a population of new users of tamoxifen, which resulted in comparable baseline characteristics of the women between exposure groups even before propensity score matching. This design helps mitigate confounding by both measured and unmeasured characteristics. No previous studies have directly compared the effect of SSRIs that are potent inhibitors versus other SSRIs on mortality among tamoxifen users. In addition, by creating two separate study cohorts distinguished by whether women were exposed first to tamoxifen or SSRIs, we were further able to minimize potential 
confounding by indication as some women are prescribed SSRIs for treatment of adverse effects of tamoxifen, such as hot flashes, whereas others are prescribed SSRIs to treat depression and anxiety related disorders. ${ }^{2021}$ Separation of the two cohorts ensured balance in the pattern of SSRI use between exposure groups. Finally, by combining multiple databases, including information for women covered through both commercial and publically funded health insurance, we were able to study a much larger population than any previous study, and we expect our results to be generalizable to the US population.

\section{Limitations}

Our study also has limitations. Information on cause of death was not available in the study databases. Therefore, we could not assess breast cancer specific mortality as an outcome. However, we conducted a sensitivity analysis among the subset of women with a previous diagnosis of breast cancer as breast cancer mortality is the most common cause of death among women with breast cancer. ${ }^{22}$ We also conducted a sensitivity analysis excluding women with stage 0 breast cancer. The results of both of these analyses were consistent with our main findings. Moreover, a meta-analysis of data from 21457 women in 20 trials found a $22 \%$ reduction in all cause mortality with tamoxifen. ${ }^{6}$ Therefore, we would expect to see an effect on all cause mortality if the interaction had a clinically meaningful impact. Another limitation is that, as with any claims based observational study, we cannot rule out potential residual confounding by smoking, obesity, and socioeconomic status. Given that we restricted our analysis to a fairly homogenous (characteristics were similar between exposure groups before matching) population of only new users of tamoxifen concomitantly exposed to SSRIs, however, we do not expect the prevalences of these unmeasured confounders to differ substantially between the exposure groups. Further, we do not expect that prescribers preferentially select SSRIs based on breast cancer prognosis. Additionally, mean follow-up in our study cohort was 2.4 years, limiting the conclusions that we can draw about the long term safety of concomitant use of tamoxifen and an SSRI.

It is also possible that information on death outside hospital assessed after 1 November 2011 for patients in the Optum Research Database might have been incomplete because, beginning in November 2011, the Social Security Administration excluded information for about $4.5 \%$ of deaths when information was available only from the states and not also from their other sources of death information. While this could have resulted in fewer outcome events, however, we expect that it would be non-differential between exposure groups, and it would not affect the specificity of the outcome definition. Another limitation inherent in the use of claims data is that we did not have information on blood metabolites of tamoxifen, and we cannot be sure that women in the study cohort actually took their dispensed drugs. Also, in our primary analysis, women remained under observation even if they no longer concomitantly used both drugs during follow-up. While this misclassification can produce bias towards the null, it allowed us to include outcomes that could have been affected by concomitant exposure even if they occurred much later. Nevertheless, results were similar in an as treated analysis, which would be less susceptible to misclassification of exposure. Finally, while claims data have been found to identify women with breast cancer with high specificity, ${ }^{23} 24$ their ability to distinguish breast cancer stages III/IV from I/II is limited. ${ }^{25}$ We would, however, expect that any misclassification would have minimal impact on the internal validity of our findings because of the similarity of women between exposure groups, even before propensity score matching.

\section{Conclusion}

In conclusion, in a large population of women treated with tamoxifen, use of potent CYP2D6 inhibiting SSRIs versus other SSRIs did not increase the risk of death from all causes. In clinical practice, SSRI inhibition of CYP2D6 does not seem to reduce the effectiveness of tamoxifen.

Contributors: MMD and JJG conceived and design the study. JJG obtained the funding. All authors analyzed and interpreted data. MMD drafted the article, which was revised critically for important intellectual content by all authors, who approved the final version for publication. All authors had full access to all of the data (including statistical reports and tables) in the study and can take responsibility for the integrity of the data and the accuracy of the data analysis. MMD and JJG are guarantors.

Funding: This study was funded by the Agency for Healthcare Research and Quality (R01HS023122 (JJG)). The sponsor had no influence on the design and conduct of the study; collection, management, analysis, and interpretation of the data; and preparation, review, or approval of the manuscript.

Competing interests: All authors have completed the Unified Competing Interest form at www.icmje.org/coi_disclosure.pdf (available on request from the corresponding author) and declare: KB is supported by a training grant from Takeda through Harvard T.H. Chan School of Public Health; JJG is principal investigator of a grant from Novartis Pharmaceuticals Corporation to the Brigham and Women's Hospital for unrelated work and is a consultant to Aetion, a software company, and to Optum

Ethical approval: This study was approved by the institutional review board (No 2014P000810) of the Brigham and Women's Hospital, Boston, MA, and data use agreements were in place.

Data sharing: No additional data available.

Transparency: The lead authors affirm that the manuscript is an honest, accurate, and transparent account of the study being reported; that no important aspects of the study have been omitted; and that any discrepancies from the study as planned (and, if relevant, registered) have been explained.

This is an Open Access article distributed in accordance with the Creative Commons Attribution Non Commercial (CC BY-NC 3.0) license, which permits others to distribute, remix, adapt, build upon this work non-commercially, and license their derivative works on different terms, provided the original work is properly cited and the use is noncommercial. See: http://creativecommons.org/licenses/by-nc/3.0/.

Torre LA, Bray F, Siegel RL, Ferlay J, Lortet-Tieulent J, Jemal A. Global cancer statistics, 2012. CA Cancer J Clin 2015;65:87-108. doi:10.3322/caac. 21262 .

2 Siegel RL, Miller KD, Jemal A. Cancer statistics, 2015. CA Cancer J Clin 2015:65:5-29 doi:10.3322/caac 21254

3 Goodwin JS, Zhang DD, Ostir GV. Effect of depression on diagnosis, treatment, and survival of older women with breast cancer. J Am Geriatr Soc 2004;52:106-11. doi:10.1111/i.1532-5415.2004.52018x.

4 Hjerl K, Andersen EW, Keiding N, Mouridsen HT, Mortensen PB, Јørgensen T. Depression as a prognostic factor for breast cancer mortality. Psychosomatics 2003:44:24-30. doi:10.1176/appi. psy.44.1.24. 
5 Early Breast Cancer Trialists' Collaborative Group (EBCTCG). Effects of chemotherapy and hormonal therapy for early breast cancer on recurrence and 15-year survival: an overview of the randomised trials. Lancet 2005;365:1687-717. doi:10.1016/S0140-6736(05)66544-0.

6 Davies C, Godwin J, Gray R, et al. Early Breast Cancer Trialists' Collaborative Group (EBCTCG). Relevance of breast cancer hormone receptors and other factors to the efficacy of adjuvant tamoxifen: patient-level meta-analysis of randomised trials. Lancet 2011;378:77184. doi:10.1016/S0140-6736(11)60993-8

7 Desta Z, Ward BA, Soukhova NV, Flockhart DA. Comprehensive evaluation of tamoxifen sequential biotransformation by the human cytochrome P450 system in vitro: prominent roles for CYP3A and CYP2D6.J Pharmacol Exp Ther 2004:310:1062-75. doi:10.1124/jpet.104.065607.

8 Lim YC, Li L, Desta Z, et al. Endoxifen, a secondary metabolite of tamoxifen, and 4-OH-tamoxifen induce similar changes in global gene expression patterns in MCF-7 breast cancer cells. J Pharmacol Exp Ther 2006:318:503-12. doi:10.1124/jpet.105.100511.

9 Dusetzina SB, Alexander GC, Freedman RA, Huskamp HA, Keating NL. Trends in co-prescribing of antidepressants and tamoxifen among women with breast cancer, 2004-2010. Breast Cancer Res Treat 2013;137:285-96. doi:10.1007/s10549-012-2330-z.

10 Binkhorst L, Bannink M, de Bruijn P, et al. Augmentation of endoxifen exposure in tamoxifen-treated women following SSRI switch. Clin Pharmacokinet 2016:55:249-55. doi:10.1007/s40262-015-0315-X.

11 Kelly CM, Juurlink DN, Gomes T, et al. Selective serotonin reuptake inhibitors and breast cancer mortality in women receiving tamoxifen: a population based cohort study. BMJ 2010;340:c693. doi:10.1136/ bmj.c693.

12 Hennessy S, Leonard CE, Gagne JJ, et al. Pharmacoepidemiologic Methods for Studying the Health Effects of Drug-Drug Interactions. Clin Pharmacol Ther 2016:99:92-100. doi:10.1002/cpt.277.

13 Gagne JJ, Glynn RJ, Avorn J, Levin R, Schneeweiss S. A combined comorbidity score predicted mortality in elderly patients better than existing scores. J Clin Epidemiol 2011;64:749-59. doi:10.1016/j. jclinepi.2010.10.004

14 Gagne JJ, Choudhry NK, Kesselheim AS, et al. Comparative effectiveness of generic and brand-name statins on patient outcomes: a cohort study. Ann Intern Med 2014:161:400-7. doi:10.7326/M13-2942.

15 Burstein HJ, Temin S, Anderson H, et al. Adjuvant endocrine therapy for women with hormone receptor-positive breast cancer: american society of clinical oncology clinical practice guideline focused update. J Clin Oncol 2014;32:2255-69. doi:10.1200/JC0.2013.54.2258.
16 Cronin-Fenton DP, Damkier P, Lash TL. Metabolism and transport o tamoxifen in relation to its effectiveness: new perspectives on an ongoing controversy. Future Oncol 2014;10:107-22. doi:10.2217| fon.13.168.

17 Higgins MJ, Stearns V. Pharmacogenetics of endocrine therapy for breast cancer. Annu Rev Med 2011;62:281-93. doi:10.1146/ annurev-med-070909-182545.

18 Decensi A, Robertson C, Viale G, et al. A randomized trial of low-dose tamoxifen on breast cancer proliferation and blood estrogenic biomarkers. / Natl Cancer Inst 2003:95:779-90. doi:10.1093/ jnci/95.11.779.

19 Kisanga ER, Gjerde J, Guerrieri-Gonzaga A, et al. Tamoxifen and metabolite concentrations in serum and breast cancer tissue during three dose regimens in a randomized preoperative trial. Clin Cancer Res 2004;10:2336-43. doi:10.1158/1078-0432.CCR-03-0538.

20 Hall E, Frey BN, Soares CN. Non-hormonal treatment strategies for vasomotor symptoms: a critical review. Drugs 2011;71:287-304. doi:10.2165/11585360-000000000-00000.

21 Shams T, Firwana B, Habib F, et al. SSRIs for hot flashes: a systematic review and meta-analysis of randomized trials. J Gen Intern Med 2014:29:204-13. doi:10.1007/s11606-013-2535-9.

22 PatnaikJL, Byers T, DiGuiseppi C, Dabelea D, Denberg TD. Cardiovascular disease competes with breast cancer as the leading cause of death for older females diagnosed with breast cancer: a retrospective cohort study. Breast Cancer Res 2011;13:R64. doi:10.1186/bcr2901.

23 Warren JL, Feuer E, Potosky AL, Riley GF, Lynch CF. Use of Medicare hospital and physician data to assess breast cancer incidence. Med Care 1999;37:445-56. doi:10.1097/00005650-199905000-00004.

24 Nattinger AB, Laud PW, Bajorunaite R, Sparapani RA, Freeman JL. An algorithm for the use of Medicare claims data to identify women with incident breast cancer. Health Serv Res 2004;39:1733-49. doi:10.1111/j.1475-6773.2004.00315.x

25 Smith GL, Shih YC, Giordano SH, Smith BD, Buchholz TA. A method to predict breast cancer stage using Medicare claims. Epidemiol Perspect Innov 2010;7:1. doi:10.1186/1742-5573-7-1.

Appendix 1: Supplementary information Appendix 2: Supplementary tables A-J 